\title{
Editorial
}

\section{Avances en la investigación sobre la dislexia evolutiva: diversidad, especificidad e intervención}

\section{Advances in Developmental Dyslexia Research: Diversity, Specificity and Intervention}

\author{
Willy Serniclaes y Juan L. Luque
}

Disponible online 31 de agosto de 2011

El objetivo de este número especial ha sido presentar una perspectiva necesariamente limitada pero significativa sobre los avances que se vienen produciendo desde hace varias décadas en la investigación sobre la dislexia evolutiva. Los artículos publicados combinan revisiones de enorme valor teórico junto con aportaciones de carácter netamente aplicado. Mientras que los artículos de Sprenger-Charolles y también el de Pernet, Dufor y Démonet abordan la diversidad de la dislexia desde diferentes perspectivas, el artículo de Serniclaes sigue una estrategia opuesta, presentando la percepción alofónica como el déficit específico de la dislexia. Explicar la variabilidad y la especificidad de la dislexia constituye un auténtico desafío para la investigación científica actual. Completan este monográfico cuatro aportaciones de carácter netamente aplicado que se han ordenado con cierta intención didáctica. Las dos primeras relacionadas con el diagnóstico. Carrillo, Alegría, Miranda y Sánchez presentan, parcialmente, una batería de evaluación de la dislexia en español, mientras que Luque, Bordoy, Giménez, LópezZamora y Rosales defiende el valor diagnóstico de un conjunto de pruebas relativas a la percepción del habla. Los dos últimos artículos representan, sin duda, aportaciones muy novedosas en el contexto de la intervención sobre las dificultades de aprendizaje de la lectoescritura. Jiménez, Baker, Rodríguez, Crespo, Artiles y Afonso, presentan un sistema de Respuesta a la Intervención aplicado en el contexto del sistema educativo público de la Comunidad de Canarias. Por su parte, Gómez, Defior y Serrano presentan detalladamente el fundamento y estructura de un programa de intervención en la fluidez lectora, un objeti- vo escasamente representado en la bibliografía en español. Veamos a continuación estas aportaciones con algo más de detalle.

Sprenger-Charolles aborda la diversidad de los perfiles disléxicos desde una perspectiva interlingüística. Los perfiles superficial y fonológico procedentes del modelo de doble ruta se utilizan para buscar diferencias entre lenguas con distinta transparencia ortográfica (inglés, francés y español). Tras hacer una revisión crítica de la metodología, la autora concluye en la ventaja del método clásico sobre el método de regresión, dada la menor fiabilidad de este último. También, se defiende que utilizar medidas tanto de exactitud como de velocidad es necesario para revelar el déficit de lectura de los disléxicos. En condiciones metodológicas óptimas, con el método clásico y considerando tanto la exactitud como la velocidad, lo que resulta es el predominio de los perfiles mixtos, seguidos de los perfiles fonológicos y superficiales, con mínimas diferencias entre lenguas. Sin embargo, en condiciones menos óptimas, prevalecen los perfiles disociados y la proporción entre perfiles fonológicos y superficiales varía entre lenguas. La autora concluye a favor de un déficit predominantemente fonológico, sin límites claros entre subtipos, lo que resulta ampliamente estable entre lenguas. Esta conclusión está muy cercana a la alcanzada por Ziegler al final de su estudio. Luego cabe preguntarse, ¿no estaremos volviendo a la unicidad e invarianza de los trastornos específicos de la lectura? O, más en línea con el artículo de Pernet y cols., ¿nos falta un método suficientemente fiable y sensible capaz de revelar diferencias definidas entre los fenotipos y los contextos lingüísticos? 
La multiplicidad es también el leitmotiv del artículo de Pernet, Dufor y Démonet. La idea que fluye a través de las líneas de este artículo es que deberíamos ser conscientes de la paradoja entre lo que se supone que es un déficit de lectura "puro", siguiendo la definición de la dislexia, y los múltiples déficits que están asociados a este trastorno. La pregunta que surge podría ser formulada en estos términos: ¿cómo podría un déficit de lectura puro enfrentarse con el hecho de que tres familias diferentes de déficits (fonológicos, viso-atencionales y de memoria-aprendizaje), cada uno subdividido en diferentes familias (p.e. para el déficit fonológico: las explicaciones auditiva y alofónica), se hayan mostrado más o menos exitosamente relacionados con la dislexia? Los autores señalan que los desórdenes fonológicos, casi los más comunes, están sólo presentes en el $40 \%$ de los participantes según una compilación de 10 artículos recientes. Respecto a las posibles respuestas, tres propuestas diferentes están en la agenda de los autores: (1) relajar el criterio para definir la dislexia permitiendo los déficits asociados; (2) investigar cada posible déficit asociado; (3) buscar fenotipos diferentes a partir de datos cerebrales. Pongamos estas propuestas en un marco de trabajo más general. Es una buena idea impulsar estudios que incluyan no sólo participantes con problemas específicos de lectura sino también aquellos con otros déficits (p.e. sordera, desórdenes de atención, autistas de alto nivel, síndrome de Williams...). Utilizar este paradigma de "banda ancha" debería permitir no sólo poner de manifiesto diferencias dentro del mismo desorden clínico (p.e. dentro de la dislexia) sino también puntos comunes entre trastornos (p.e. entre disléxicos y autistas de alto nivel). Nótese sin embargo, que en el estado actual del asunto, el paradigma de banda ancha no requiere redefinir los diferentes trastornos clínicos ¿Investigar sistemáticamente los déficits fonológicos, visuales, auditivos? Porque no, pero pensemos dos veces. El coste/beneficio de una investigación de múltiples pistas será prohibitivo si los diferentes criterios utilizados no están claramente relacionados con hipótesis bien definidas. Finalmente, los datos cerebrales a menudo revelan más de lo que pueden mostrar los datos conductuales (véase el artículo de Serniclaes) y podrían incluso mostrarse muy útiles para propósitos taxonómicos.

Por su parte, el artículo de Serniclaes presenta una revisión de la teoría del modo alofónico. Uno de los retos de la investigación actual es determinar la relación exacta entre la causa hipotética y las dificultades en el aprendizaje de la lectura. Es ampliamente admitido que la conciencia fonológica identifica la causa próxima de las dificultades de aprendizaje de la lectura. Sin embargo, tan pronto como se afirmó que el déficit disléxico estaba causado por una pobre representación de los fonemas, su almacenamiento y/o procesamiento, aparecieron hipótesis relativas a la percepción de los sonidos o fonemas (Tallal, 1980). Sin embargo, la llamada hipótesis del procesamiento auditivo rápido no se ha mostrado específica de la dislexia, ni ha conseguido detallar cuál es la conexión entre el déficit auditivo y el aprendizaje de la lectura (Ramus, 2003). Tampoco la constatación reiterada de diferencias relativas a la percepción categorial entre controles y disléxicos había permitido progresar en este sentido (Serniclaes et al, 2004). El principal activo de la hipótesis alofónica es precisamente este: ofrece una explicación directa que conecta el déficit, en este caso la percepción alofónica, con la dificultad de aprendizaje de las reglas de conversión. El modo de percepción alofónico sería la consecuencia de dificultades en la transición entre los límites fonéticos universales y los propios de la lengua materna. La permanencia en activo de algunas de las categorías universales afectaría directamente al establecimiento de correspondencias entre sonidos y letras. En concreto, correspondencias que normalmente son entre dos sonidos y dos letras, serían de tres sonidos a dos letras (/b-p-

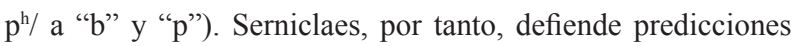
muy concretas y falsables que en esta nueva revisión alcanzan una nueva formulación: mientras que los diversos déficit en la precisión perceptiva no son específicos de la dislexia, la percepción alofónica sí. Tal y como defiende el autor el interés teórico en los problemas de percepción categorial no está en que los disléxicos muestren debilidad en distinguir entre categorías, sino en que muestran una mejor discriminación dentro de las categorías. El artículo se completa con dos interesantes aportaciones. Primero, la identificación del área premotora izquierda como la zona crucial donde se procesan fonemas versus alofónos. Estos estudios han puesto de manifiesto además otro aspecto de interés: aunque las medidas conductuales tienen dificultades para captar regularmente la alofonía, los registros de la actividad cerebral complementarios muestran el fenómeno. Finalmente, se revisan los estudios que han intervenido sobre la percepción fonémica concluyendo que existen pruebas de su efecto para remediar la dislexia.

Carrillo, Alegría, Miranda y Sánchez nos presentan parte del sólido trabajo que vienen desarrollando para construir una herramienta fundamentada y sencilla con la que detectar las dificultades específicas de la lectura y realizar el diagnóstico de la dislexia evolutiva. Tal y como nos explican los autores la batería posee tres herramientas básicas para aplicar de forma secuencial. En primer lugar, una prueba de detección de gran sencillez y economía, dado que se aplica a grupos y su duración es de cinco minutos. Esta prueba permite identificar hasta un $96 \%$ de alumnos que posteriormente mostraran retraso en los mecanismos de identificación de palabras. Para valorar el retraso o alteración de tales mecanismos, la batería DIS-ESP dispone de un conjunto seleccionado de tareas relativas a los mecanismos fonológicos y ortográficos implicados en la lectura de palabras. Estas pruebas han mostrado en este estudio que una vez que la experiencia lectora empieza a tener peso, la mayoría de los alumnos con retraso lo manifiestan en ambos mecanismos, fonológico y ortográfico. Hasta un $85 \%$ de los alumnos con dificultades de los cursos de cuarto y sexto presentan un perfil de retraso mixto. Esta prevalencia de los perfiles mixtos es intepretada por los autores a partir de la hipótesis 
de Share (1995): la consolidación del mecanismo fonológico permitiría el autoaprendizaje y éste fortalecería progresivamente la representación ortográfica de las palabras. Finalmente, la prevalencia de dificultades lectoras detectadas está cercana a las halladas en estudios nacionales e internacionales, aunque se advierte que no todos los casos serán finalmente diagnosticados como dislexia. Para llegar a un diagnóstico de dislexia se recomienda utilizar un tercer conjunto de herramientas: un conjunto de pruebas fonológicas -donde no interviene la lectura- que representan las distintas áreas donde se ha verificado el déficit fonológico básico (Wagner y Torgersen, 1987).

El estudio de Luque, Bordoy, Giménez, López-Zamora y Rosales se plantea desarrollar y testar un conjunto de pruebas relativas a la percepción del habla junto con un subconjunto de las medidas que de éstas pueden derivarse. La idea es replicar algunos hallazgos significativos de la bibliografía especializada para después estudiar la utilidad de las medidas como criterio de desviación con respecto a muestras de sujetos controles igualados en edad o igualados en nivel lector. Se argumenta de forma ecléctica que independientemente de si los déficits detectados con estos procedimientos son o no específicos de la dislexia, estas medidas presentan un enorme valor diagnóstico. Pueden ayudar a detectar la comorbilidad entre el trastorno específico del lenguaje y la dislexia. Pueden detectar dificultades de precisión perceptiva $\mathrm{u}$ otras debilidades perceptivas presentes en disléxicos y en otros trastornos. Finalmente, pueden incluso detectar el déficit alofónico, que Serniclaes en este volumen defiende como específico de la dislexia, aunque en el estudio presente no se desarrolle esta capacidad. Por tanto, los autores concluyen que estos instrumentos pueden ayudar a ponderar la desproporción entre habilidades fonológicas y el resto de capacidades cognitivas que supuestamente los disléxicos tienen intactas. Del mismo modo, son de gran interés para el estudio de los trastornos específicos del lenguaje, los patrones comórbidos, y, en general, para estudiar síntomas asociados a perfiles disléxicos individuales.

Lo sistemas de Respuesta a la Intervención que se han extendido por los EE.UU durante la última década son herederos directo de una idea más general: la práctica educativa debe basarse en el conocimiento científico (event-based practise). Mientras que esta filosofía está ampliamente aceptada en el área de salud, constituye una auténtica novedad en nuestro sistema educativo la experiencia que se está llevando a cabo en la Comunidad de Canarias. Los sistemas de Respuesta a la Intervención son una alternativa de carácter institucional al problema de la detección precoz y tratamiento adecuado de las dificultades de aprendizaje. Estos sistemas implican tanto a instituciones de investigación, como a la administración educativa y sus agentes, los colegios. Dentro de esta colaboración un instrumento básico es el sistema de evaluación continua del desempeño escolar. Estas herramientas permiten la detección precoz y evaluación permanente de un conjunto de habilidades referidas directamente con el curriculum base de la enseñanza de la lectura.
Afortunadamente, la Universidad de Oregón desarrolló estos instrumentos tanto para alumnos anglosajones como hispanohablantes. Conocidos con las siglas IDEL (Indicadores Dinámicos del Éxito en la Lectura; Good, Baker, Knutson y Watson, 2006), estas pruebas han permitido una fluida colaboración y adaptación de estos procedimientos a la Comunidad de Canarias. Garantizada la alta calidad científica en la formación de los profesionales implicados, el sistema permite tomar decisiones educativas basadas en información objetiva y dinámicamente contrastada, ajustando la intensidad de los tratamientos y los recursos materiales y humanos disponibles. Finalmente, en el artículo se presentan y comentan los baremos específicos para la Comunidad Canaria, lo que ejemplifica otra característica de los sistemas RtI: la posibilidad de comparar la ejecución de un individuo o escuela tomando como referencia diferentes criterios, nacionales, provinciales, comarcales, de distrito o incluso tan sólo la propia clase.

El artículo de Gómez, Defior y Serrano sobre fluidez lectora es novedoso en al menos dos sentidos. Es heredero del mismo impulso por transferir el conocimiento científico a la práctica educativa. Este objetivo se concretó en el National Reading Panel (NRP, 2000). Los resultados del NRP destacaron cinco áreas fundamentales de intervención: conciencia fonémica, principio alfabético, vocabulario, fluidez y comprensión lectora. Aunque existen propuestas para trabajar la fluidez lectora en español ya publicadas, estas no han sometido su eficacia a contrastación empírica. Existe una distancia fundamental entre los programas que están guiados cientificamente con aquellos cuya eficacia está probada cientificamente. Se presenta en este número tan sólo la estructura básica del programa, pero pronto podrán leerse los resultados científicamente contrastados. El artículo, además, ilustra de forma detallada el proceso de fundamentación científica que debe seguirse durante el desarrollo de un programa de intervención. Es, en este sentido, un ejemplo de una de las etapas fundamentales en la transferencia del conocimiento científico a la práctica educativa. Pero además de estos aspectos metodológicos o formales, el programa integra un trabajo específico que recorre desde unidades subléxicas hasta el trabajo sobre el texto, beneficiándose de los avances obtenidos a partir de dos técnicas principalmente, los métodos de lectura acelerada y los de lectura repetidas. Estamos, por tanto, ante un modelo a imitar en la construcción de herramientas de intervención científicamente fundamentadas y contrastadas.

Finalmente, recordar que este número monográfico se ha realizado con la intención de impulsar la investigación sobre la dislexia evolutiva pero también, de forma especial, con el compromiso de transferir a los agentes educativos parte del inmenso corpus de conocimientos científicos del que pueden disponer en sus prácticas de evaluación, diagnóstico e intervención en las dificultades de aprendizaje específicas de la lectura. Esperamos haber contribuido mínimamente con estos dos grandes objetivos. 


\section{Referencias}

1. Good, R. H., Baker, D. L. Knutson, N. y Watson, J. M. (Eds.). (2006). Indicadores dinámicos del éxito en la lectura (7a ed.). Eugene, OR: Dynamic Measurement Group, Inc. Acceso: http://dibels.uoregon.edu/

2. National Reading Panel (2000). Teaching children to read: An evidence-based assessment of the scientific research literature on reading and its implications for reading instruction: Reports of the subgroups. Bethesda, MD: National Institute of Child Health and Human Development. Disponible en: http://www.nationalreadingpanel.org/

3. Ramus, F. (2003). Developmental dyslexia: specific phonological deficit or general sensorimotor dysfunction? $\mathrm{Cu}$ rrent Opinion in Neurobiology, 13, 1-7.

4. Serniclaes, W., Van Heghe, S., Mousty, Ph., Carré, R., \& Sprenger-Charolles, L. (2004). Allophonic mode of speech perception in dyslexia. Journal of Experimental Child Psychology, 87, 336-361. http://dx.doi.org/10.1016/j. jecp.2004.02.001
5. Share, D.L. (1995). Phonological recoding and self-teaching: sine qua non of reading acquisition. Cognition, 55, 151-218. http://dx.doi.org/10.1016/0010-0277(94)00645-2

6. Tallal, P. (1980). Auditory temporal perception, phonics, and Reading disabilities in children. Brain Language, 9, 182-198. http://dx.doi.org/10.1016/0093-934X(80)90139$\underline{\mathrm{X}}$

7. Wagner, R. K., y Torgesen, J. K. (1987). The nature of phonological processing and its causal role in the acquisition of reading skills. Psychological Bulletin, 101(2), 192-212.

8. Ziegler, J.C., Castel, C., Pech-Georgel, C., George, F., Alario, F-X. \& Perry, C. (2008). Developmental dyslexia and the dual route model of reading: Simulating individual differences and subtypes. Cognition, 107, 151-178. http:// dx.doi.org/10.1016/j.cognition.2007.09.004 